\title{
"POR LAS MALEFACTURAS QUE SE FAZIAN ENTRE LAS TIERRAS DE ÇAPUERTOS E LA TIERRA DE LABORT". LA COMPLEJA DEFINICIÓN DE LA FRONTERA MEDIEVAL Y SU CONTROL SEGÚN EL EJEMPLO NAVARRO DE ULTRAPUERTOS (SIGLOS XIII Y XIV)
}

\author{
Susana Aparicio Rosillo ${ }^{2}$
}

\section{RESUMEN}

Este artículo presenta la realidad de la frontera ultraportana de Navarra en los siglos plenomedievales, una línea fronteriza que ha sido poco estudiada hasta ahora. Sin embargo, su importancia resulta innegable a la luz de los esfuerzos de la monarquía navarra por controlar este espacio y asentar su poder sobre él, a través de herramientas diversificadas, como el control administrativo y recaudatorio, las fortalezas reales y la fundación de nuevos núcleos de población, entre otras cosas.

Palabras Clave: frontera, Ultrapuertos, administración, fortalezas, bastidas.

\section{RESUMÉ}

Cet article nous apprend la complexité de la frontière de Navarre en d'Outre-Ports pendant le Plein Moyen Âge. Cette frontière a été l'objet de peu d'études jusqu'aujourd'hui. Cependant, son importance reste incontestable à l'égard des efforts réalisés par les souverains navarrais pour contrôler ce territoire et pour affirmer son pouvoir là-bas. Ils ont profité d'outils très divers pour y parvenir, et parmi eux on peut nommer l'administration du territoire et des impôts, les forteresses royales et la fondation de nouveaux bastides.

Mots Clés: frontière, Outre-Ports, administration, forteresses, bastides.

Fecha de recepción: 10 de febrero de 2011. Fecha de aceptación: 9 de marzo de 2011

1 Juan CARRASCO et al: Acta Vectigalia Regni Navarre, tomo VI, ed. Gobierno de Navarra, Pamplona, 2001, p. 199.

2 Universidad Pública de Navarra. Email: susana_apar@yahoo.es. 
A la hora de definir las fronteras del reino de Navarra en la época medieval ${ }^{3}$, se tiende a pensar en primer lugar en la frontera con Castilla, definida por Álava y Guipúzcoa, que en un pasado no tan lejano - anterior a 1200 - habían formado parte de este reino, aunque en ese momento era conocida como "frontera de los malhechores". Fue definida de este modo por las propias fuentes contemporáneas a causa de su conflictividad latente y las constantes escaramuzas procedentes de uno y otro lado, y ha sido estudiada en profundidad por varios destacados historiadores ${ }^{4}$. La segunda realidad en la que se piensa es los límites del reino con Aragón, más definidos y caracterizados por un menor nivel de enfrentamientos ${ }^{5}$. Sin embargo ¿qué ocurría con el apéndice pirenaico del reino navarro, las tierras de Ultrapuertos, que definían a su vez una línea fronteriza?

Estos territorios constituían desde un principio una frontera de naturaleza compleja, puesto que lindaban con dos poderes tan relevantes como el del rey de Inglaterra y el del vizconde de Béarn. Incluso la definición del origen de la ocupación navarra en este espacio se revela como dificultosa, ya que se desconocen las causas últimas de la primera implantación navarra más allá de los Pirineos. En cualquier caso, a finales del siglo XII los navarros ya controlaban la tierra de Cisa, a la que más tarde se le irían añadiendo el valle de Osses y el vizcondado de Baigorry, a través de la adhesión de su titular a la causa navarra. Posteriormente obtuvieron un dominio efectivo sobre el valle de Arberoa y la tierra de Mixa tras un enfrentamiento militar con el titular de estos territorios, el vizconde de Tartas, que finalmente comprometería sus tierras en homenaje con el monarca navarro. En esta misma contienda se arrebataron a los ingleses las parroquias de Iholdy y Armendariz. De este modo a mediados del siglo XIII quedaron configurados los territorios de Ultrapuertos tal y como se conocían en la época medieval. Por añadidura, el rey de Navarra contaba con la eventual fidelidad del señor de Agramont y del vizconde de Soule, cuyas posesiones eran piezas clave para el entramado fronterizo navarro, puesto que los agramonteses lindaban con las parroquias inglesas, mientras que las tierras souletinas actuaban como barrera frente al vizconde de Béarn.

3 En cuanto a estudios sobre frontera, podemos destacar el seminario de la Universidad de Zaragoza sobre Las sociedades de frontera en la España medieval o bien "Espacios y organización social" en Estudios de Geografía e Historia, n 4, Madrid, 1989; el trabajo de Juan REGLÁ: Francia, la Corona de Aragón y la frontera pirenaica, ed. CSIC, Madrid, 1951 o los estudios de Philippe SÉNAC: Frontières et espaces pyrénéens au Moyen Âge, Perpignan, 1992.

4 Se pueden citar, entre otros, la monografía de J. Luis ORELLA y J. Ángel ACHÓN: Guipúzcoa y el Reino de Navarra en los siglos XIII-XV: relaciones, intereses y delimitación de la frontera, ed. Univ. de Deusto, San Sebastián, 1998 y los artículos de J. Ramón DÍAZ DE DURANA, J. Andoni FERNÁNDEZ DE LARREA: "La frontera de los malhechores: bandidos, linajes y villas entre Álava, Guipúzcoa y Navarra durante la Baja Edad Media" en Studia Historica. n 23, 2005, p. 171-205; J. Angel ACHÓN: "Los intereses banderizos en la definitiva configuración de la frontera entre Guipúzcoa y el Reino de Navarra” en Príncipe de Viana. Anejo, n 8, 1988, p. 257-266; Iñigo MUGUETA: “Acciones bélicas en Navarra: La frontera de los malhechores (1321-1335)" en Príncipe de Viana, $\mathrm{n}^{\circ} 219,2000$, p. 49-78 o Fco. Javier ZABALO: "El acoso de guipuzcoanos y alaveses a los ganaderos navarros: la frontera de los malhechores entre 1280 y 1349” en Príncipe de Viana, n 234,2005, p. 53-110.

5 Joaquín SALCEDO: "Historia convergente de Aragón y Navarra" en Iacobus: revista de estudios jacobeos y medievales, $\mathrm{n}^{\circ}$ 15-16, 2003, p. 99-112 o M Carmen LACARRA: "Intercambios artísticos entre Navarra y Aragón durante el siglo XV” en Príncipe de Viana. Anejo, n 11, 1988, p. 279-296. 
Las fronteras del territorio ultraportano requerían pues una atención especial por parte de los agentes reales navarros, de cara a obtener una dominación efectiva del espacio, y del mismo modo evitar las incursiones desde el otro lado de la línea divisoria. Los instrumentos de control que se aplicaron fueron principalmente el establecimiento de unas demarcaciones administrativas bien definidas, que contaban con un rosario de oficiales regios dedicados a la gestión política y fiscal del territorio ${ }^{6}$. En segundo lugar, el control de las mercancías que pasaban por la zona fronteriza se logró mediante el establecimiento de fuertes aduanas y peajes, como se comentará más adelante.

Por otro lado, el soberano se apoyaba en el sometimiento de la nobleza local para sostener la presión militar y establecer una línea de fortalezas que vigilaran las fronteras del reino en Ultrapuertos. Sin embargo, a cambio de este apoyo debía hacer frente también a las constantes actividades "poco lícitas" de estos mismos nobles, los enfrentamientos privados, el contrabando, el establecimiento de peajes no autorizados en sus tierras y el ataque a los mercaderes y peregrinos que cruzaban las vías de comunicación que ellos controlaban?.

Los monarcas navarros también se centraron en la creación de nuevos enclaves dotados de privilegios que favorecían la afluencia de población, en concreto en las localidades de Saint Palais y Labastide Clairence - en ambos extremos del mapa - que se analizarán más adelante. Por último, el control de Roncesvalles sobre ciertos espacios ultraportanos y labourdinos como la encomienda de Bidarray ${ }^{8}$ contribuyó de la misma manera a afianzar la presencia navarra sobre dicho territorio.

De hecho, el hospital navarro de Roncesvalles contaban con encomiendas dependientes a lo largo de la zona pirenaica. Entre ellas se pueden citar la de Bonloc en Labourd, Bidarray, San Juan el Viejo, Bussunaritz, Mocosail, San Miguel y Ordiarp - situada en Soule, muy cercana a Mauleón - entre otras, además de toda una serie de dotaciones estratégicamente localizadas a lo largo de la zona aquitana, notablemente en los nodos de comunicación, con todo un rosario de capillas e iglesias dependientes ${ }^{9}$. Como ejemplo, se podrían citar las iglesias que aparecían en un documento de 1213: Santa Eulalia de Isturiz, en territorio labourdino, del mismo modo que Lekhorne y Ayherre, y la de San Esteban de Baigorry, en pleno corazón del vizcondado del mismo nombre ${ }^{10}$. Sin embargo, no se va a profundizar en las estrategias de implantación de los establecimientos religiosos en la

6 Estudiados precisamente en la tesis de Susana HERREROS: Las tierras navarras de Ultrapuertos (siglos XII-XVI), ed. Gobierno de Navarra, Pamplona, 1998.

7 Mateo PARIS: Chronica Maiora, tomo V, p. 338: "Los gascones, los cuales eran grandes ladrones, expoliaban a los mercaderes y peregrinos que pasaban por allí, teniendo refugio en antiguas cavernas de ladrones, por ejemplo en la peña inaccesible de Agramont, la cual fortificaron con un castillo...." Sin embargo, este comportamiento se reproducía también en otras zonas de frontera, como nos recuerda en su artículo M. Ángel LADERO: "Sobre la evolución de las fronteras medievales hispánicas" en Identidad y representación de la frontera en la España medieval (siglos XI-XIV), ed. Casa de Velázquez, Madrid, 2001, p. 48.

8 La encomienda de Bidarray aparecía citada en varios documentos reales, y en algún caso se le emiten beneficios, por ejemplo la remisión de una pena por injurias a los representantes navarros al abad de Bidarray en 1381 (AGN, Comptos, caj. 44, nº 3).

9 Fermín MIRANDA: Roncesvalles, trayectoria patrimonial, ed. Gobierno de Navarra, Pamplona, 1993 p. 99.

10 AGN, Códices, C. 1, fol. 185. 
zona ultrapirenaica, puesto que lo que se está tratando en este trabajo serían las estrategias regias de control de la frontera.

Uno de los mecanismos ya mencionados, esgrimidos por los monarcas navarros para asegurar su presencia en las tierras ultrapirenaicas, era el control del flujo de mercancías que atravesaban esta zona. De todos es sabido que las instituciones reales medievales obtenían un beneficio considerable de las transacciones comerciales, entre otros mediante las imposiciones que gravaban el intercambio de bienes, además de los peajes que debían pagar los comerciantes al transportar sus mercancías. Sin embargo, si bien Ultrapuertos les ofrecía una nada desdeñable plataforma recaudatoria - debido a que se encontraba en la encrucijada de varias rutas comerciales de notable relevancia-, también es cierto que su frontera con el Labourd, una tierra de adscripción distinta y que por tanto tributaba al rey de Inglaterra, favorecía el contrabando de mercancías, eludiendo el control regio. De este modo, en la documentación navarra se pueden encontrar constantes referencias a este tipo de transacciones ilícitas y al control que intentaba establecer el monarca sobre ellas, sobre todo en el caso de ventas de $\operatorname{pan}^{11}$ o de ganado ${ }^{12}$. Así mismo, los habitantes de las tierras de Ultrapuertos intentarían también deshacerse de estas imposiciones sobre las transacciones, alegando la costumbre que tenían de comerciar con ambos lados de la frontera ${ }^{13}$.

Por el contrario, al centrarse en un marco de "legalidad", también se puede afirmar que los agentes reales estimularon el flujo de los intercambios entre las dos vertientes de esta frontera, ya que favorecía sus intereses y engrosaba las arcas del erario, como en el caso del ganado porcino. En el registro de la quinta de los cerdos en Navarra, un impuesto establecido por la trashumancia estacional del ganado a otros pastos, quedaban los movimientos de dichos animales que pasaban sin problemas desde Labourd hasta los Alduides o a la zona del Bidasoa, de Arberoa, Maya, Armendariz, Ezpeleta y Garro al Baztán, e incluso de zonas más alejadas como las bearnesas, en concreto, Oloron, hacia el valle de Aézcoa ${ }^{14}$. Del mismo modo, a mediados del siglo XIV también se localizaban los registros detallados de las mercancías - ganado y alimentos - que pasaron de Navarra a Gascuña o viceversa ${ }^{15}$. Por tanto, los intercambios continuaban siendo fluidos, al menos en cuanto a movimientos de ganado, por toda la frontera ultraportana.

Este territorio se encontraba también en un conflicto constante, ya que como se ha comentado los límites territoriales no estaban del todo definidos. Esta realidad condujo a que se produjeran enfrentamientos incluso entre regiones pertenecientes a la misma entidad ultraportana, sin entrar ya a menciona, cómo se aprovechaba la situación fronteriza

11 Asi se detalla en un documento real de 1388 “...por la vendition del pan que a seido fecha en la dicta merindat et terra d'Aillent Puertos que venia fuera de nuestro Regno, el qual nos deffendiemos et mandamos que non pagasse imposition" (AGN, Comptos, caj. 57, $\mathrm{n}^{\circ}$ 40, VI).

12 AGN, Comptos, caj. 18, $\mathrm{n}^{\circ} 128, \mathrm{X}$.

13 Así lo declaraban en Cisa a mediados del siglo XIV "Otrossi seynnor cada que nos otros de la dicta tierra de Cisa avemos mester comprar pan para nuestra provision et avemos siempre vsado et costumbrado de comprar et traer a qual do quiere qui lo podemos aun sea en el regno o fuera del regno..." (AGN, Comptos, caj. $\left.57, \mathrm{n}^{\mathrm{o}} 111, \mathrm{III}\right)$.

14 AGN, Comptos, caj. 66, no 19, I, fol. 8 r-8v. y 11 r.

15 AGN, Comptos, caj. 12, no 62. 
para realizar incursiones en los territorios ingleses que reportaban frecuentes presas de ganado o alimentos ${ }^{16}$. La propia administración real era consciente de esta realidad cuando definía los enfrentamientos entre las tierras de Osses y Cisa como si fuessen enemigos del rey et del regno ${ }^{17} \mathrm{o}$ al especificar a su baile de Mixa que no tratase a los habitantes de las tierras de Mixa y las parroquias agramontesas como si fuesen extranjeros del regno ${ }^{18}$, aunque esta indefinición se explicará con más detalle en el apartado siguiente.

\section{PROBLEMAS DE DEFINICIÓN DE CIERTOS TERRITORIOS EN LA ZONA DE ULTRAPUERTOS}

Los propios márgenes de estos territorios ultraportanos fueron los que mayor problemática generaron, debido a que su cercanía a tierras de adscripción diferente provocaba una cierta indefinición, sobre todo en sus intercambios comerciales. Así ocurría por ejemplo en el caso de zonas de Mixa, colindantes con las posesiones del señor de Agramont. No quedaba claro a qué señor debían ser adscritas, de ahí la constante repetición el documento que aparece al final de este trabajo, en el que se insistía en su inclusión dentro de los márgenes del regno de Navarra ${ }^{19}$. Este territorio constituía un punto fundamental para los dominios del rey navarro en Ultrapuertos, que conectaba directamente con la zona de Came y Escos, y lo que era más importante, con la cuenca fluvial del Bidouze, que actuaba como eje comercial en la región.

La dependencia navarra de las tierras de Mixa estaba ligada al juramento de fidelidad de los señores de Tartas, inaugurado en $1247^{20}$, y a partir de ese momento se empezó a incluir este territorio en las cuentas reales navarras ${ }^{21}$. Sin embargo, poco después comenzaron los problemas de adscripción, ya que las tierras de Ostabares, también pertenecientes al señorío de Tartas, se opusieron a los oficiales reales en 1305, alegando su dependencia de un señorío particular y no del rey²2. Del mismo modo, al cambiar la titularidad a la familia de Albret a finales del siglo XIII, la situación de estas tierras convirtió en más compleja. Esto llegó a tal punto que, a causa de la rebeldía de uno de los miembros de este clan, Bernard-Ezii — que decidió tomar partido por los ingleses - , el propio monarca franco-navarro confiscó estas tierras en 1338, que permanecieron bajo tutela directa del rey durante casi medio siglo ${ }^{23}$. Esta situación, al ser devueltas a los Albret, fue aprovechada por la población de la zona para negarse a pagar las rentas reales —en 1368 y 1370 - , con la excusa de que sólo debían rendir cuentas ante dicha familia

16 Susana APARICIO: "La violencia en Gascuña y los enfrentamientos anglo-navarros (siglos XIII y XIV)" en Huarte de San Juan. Geografía e Historia, no 14, 2007, p. 9-32.

17 “... como si fuessen enemigos del rey et del regno, aven entrado en la tierra de Osses dentro et plegados los ganados casi robados o forcados...” (AGN, Papeles sueltos, $2^{\mathrm{a}}$ serie, leg. 3, nº 89, II).

18 Ver documentación de apoyo, pieza 2.

19 Ídem.

20 J. Auguste BRUTAILS: Documents de la Chambre de Comptes de Navarre, p. 10, doc. XII.

21 En concreto, a partir de 1266, según queda constancia en Juan CARRASCO et al. Acta Vectigalia ..., tomo I.

22 Susana HERREROS: Las tierras navarras de Ultrapuertos..., p. 114.

23 Iñigo MUGUETA: "La nobleza en Navarra (siglos XIII-XIV): una identidad militar", en Iura Vasconiae, $\mathrm{n}^{\mathrm{o}}$ 4, 2007, p. 206 y la lealtad inglesa de Bernard-Ezii aparecía consignada en ADPA, E 132. 
nobiliaria ${ }^{24}$. Los problemas del poder navarro con las tierras de Albret continuaron a lo largo de todo el siglo XIV, dificultando la implantación de su poder de forma efectiva sobre este territorio.

Este no sería el único caso en el que las fronteras no se hallaban claramente definidas. La localidad de Ainhoa, situada en la zona labourdina, se encontraba en un caso similar de indefinición territorial, probablemente debido a su naturaleza de punto estratégico, situado junto al paso pirenaico secundario de Dantxarinea. Tras el enfrentamiento anglonavarro zanjado precisamente en esta parroquia en 1248, la mitad de dicho espacio quedó en manos de Navarra, pero su posesión fue contestada en varias ocasiones. Se podría citar como paradigmática la reclamación realizada en 1305 por García Arnaldo, señor de Ezpeleta, en la que solicitaba unas cartas que garantizaran la posesión de Ainhoa, puesto que las que tenía se perdieron con otros bienes en la guerra ${ }^{25}$. Los navarros insistían en que esta parroquia se encontraba dentro de su patrimonio real, mientras que los agentes del rey inglés en Labourd afirmaban que se localizaba dentro los límites de su reino, y por tanto pedía que se solucionara el pleito ${ }^{26}$. Finalmente, esta localidad quedó dentro de la obediencia inglesa.

\section{EL CONTROL ADMINISTRATIVO}

Las estrategias de los monarcas navarros para afianzar su poder sobre este territorio pasaban en primer lugar por crear unos resortes administrativos fuertes y operativos, un asunto que queda ampliamente demostrado a través de la tesis de Susana Herreros Lopetegui. En ella se demuestra que, pese a que este territorio no tuvo la misma denominación que una merindad, su recaudación era cuidadosamente controlada por la administración real. Del mismo modo, sus instituciones se encontraban centralizadas en torno al castellano de San Juan de Pie de Puerto, una villa que actuaba como cabecera del territorio.

Por otro lado, a mediados del siglo XIV las diferentes demarcaciones que integraban la zona de Ultrapuertos se distribuían de la siguiente manera: la bailía de Labastide Clairence, la de de Mixa y Ostabares, la castellanía de San Juan de Pie de Puerto y dos señoríos particulares, el de Baigorry y las tierras del señor de Agramont, cuya tributación, al ser señoríos particulares, no se realizaba directamente a los oficiales del rey.

La castellanía de San Juan de Pie de Puerto y la localidad del mismo nombre centralizaban los movimientos administrativos navarros ultrapirenaicos. Esta castellanía abarcaba las tierras que constituían el núcleo originario de la implantación navarra en el espacio del otro lado de los Pirineos. Esto es, la tierra de Cisa y el valle de Osses, a los que se les añadiría tras el conflicto zanjado en 1248 las parroquias de Iholdy y Armendariz - arrebatadas a los ingleses - y el valle de Arberoa. Las primeras menciones a este territorio, o en concreto a la demarcación de Cisa son paralelas al asentamiento navarro en estas tierras, de modo que existían referencias a un baile de Cisa desde finales del siglo XII ${ }^{27}$.

24 AGN, Registros, $\mathrm{n}^{\circ} 119$, fol. $435 \mathrm{v}$.

25 Ver documentación de apoyo.

26 Charles BÉMONT: Rôles Gascons, tomo II, doc. 1195, p. 374

27 Victor-Pierre DUBARAT, J. B. DARANATZ: Un Procès entre l'évêché de Bayonne et le monastère de Roncevaux au XIVe siècle (1332-1335), ed. Impr. du «Courrier», Bayona, 1926, p. 111. 
Los comptos navarros registraron sus aportaciones al erario real desde 1266 regularmente, aunque las atribuciones del castellano de San Juan variaron ligeramente a lo largo del tiempo, como la incorporación de un recibidor en 1351, entre otras cuestiones ${ }^{28}$.

El hecho de que en algunas ocasiones a lo largo del siglo XIV el castellano de San Juan aglutinara también otros cargos como baile de Mixa o Labastide podría indicar una preeminencia de este oficial, hasta llegar a ser una especie de "pseudo-merino", pero historiadores de la talla de Ángel Martín Duque afirman que simplemente se trataba de una coincidencia puntual, no de una estrategia regia de centralización ${ }^{29}$. Por tanto, si bien espacialmente se trataba la demarcación más amplia y mejor controlada de Ultrapuertos, no contaba con una preeminencia administrativa sobre las demás.

De las tierras de Mixa ya se ha hablado anteriormente. Sus problemas radicaban ante todo en una implantación posterior del poder navarro en la zona. Se trataba de un espacio dependiente del señor de Tartas, que juró lealtad al rey navarro, Teobaldo II, en 1247, tras un conflicto armado y la ocupación militar navarra de la zona ${ }^{30}$. Sin embargo, las posesiones de los señores de Tartas pasaron posteriormente a manos de una de las familias más destacadas de la zona labourdina, los Albret, y por tanto estas tierras se encontraban dentro de una realidad geopolítica compleja e indefinida. A pesar de todo, los agentes navarros controlaban el territorio del mismo modo que las demás demarcaciones, salvo en momentos puntuales ${ }^{31}$.

En cuanto a la bailía de Labastide Clairence, se creó tras la fundación de dicha bastida en 1312, de la que se hablará más adelante. Estas tierras se desgajaron del valle de Arberoa, perteneciente a la castellanía de San Juan, y ya contaban con un oficial real que ejercía como baile un año después de haber obtenido su fuero. Sin embargo, no es posible localizar repertorios seriados de imposiciones registrados en los Comptos reales, debido a su propio carácter de territorio exento de rentas. Sin embargo, el baile ejercía el resto de las funciones, esto es, el mantenimiento de la fortaleza asociada a este lugar - la antigua Naupeciada - y la ejecución de la justicia dentro de este territorio, reflejada en la lista de multas. Por tanto, y al igual que en los demás territorios ultraportanos, e incluso con un mayor cuidado al tratarse de uno de los puntos estratégicos fundamentales en su frontera, el monarca navarro mantenía un estrecho control sobre esta zona a través de sus oficiales ${ }^{32}$.

\section{LAS FORTALEZAS FRONTERIZAS}

Una de las características que comparten los espacios de frontera medievales es la necesidad de control visual y militar de la zona. Por tanto, lo que se establecía es toda una red de fortificaciones que ejercían como puntos estratégicos dentro del cinturón fronteri-

28 Para un mayor detalle, consultar la tesis de Susana Herreros, dedicada precisamente al estudio de los resortes administrativos de este territorio (para San Juan de Pie de Puerto, por ejemplo, a partir de la p. 158).

29 Así lo afirma también Susana HERREROS: Las tierras navarras..., p. 154.

30 AGN, Códices, C. 1, fol. 124-126.

31 Ver nota $\mathrm{n}^{\circ} 21$.

32 Para conocer una lista de los bailes de Labastide, se puede consultar la obra de Susana HERREROS, a partir de la página 163. 
$\mathrm{zO}^{33}$. Estos castillos de frontera se convirtieron en focos de atracción para la población de las zonas circundantes, que buscaba el amparo que ofrecían y las posibilidades de salida a sus productos, al situarse también como puntos de supervisión de las principales vías de comunicación.

En esta zona se puede citar como ejemplo más paradigmático el cinturón defensivo del Labourd, en cuya frontera se alineaban fortalezas de obediencia navarra que vigilaban la frontera inglesa ${ }^{34}$. Así ocurría en el caso del castillo señorial de los Belzunce, situado en la zona de Ayherre en Hasparren, cuyo emplazamiento controlaba el camino de la montaña procedente de Labastide Clairence. Dicha fortaleza se encontraba estratégicamente situada sobre una elevación, dominando todo el valle de Arberoa y las tierras circundantes. En el marco de la conflictividad imperante en este territorio, el linaje que lo controlaba, los Belzunce, formaba parte del bando navarro, y vigilaba en consecuencia los movimientos ingleses. El señor de Belzunce contaba además con una edificación fortificada de carácter secundario, probablemente una torre o una casa fuerte en la parroquia de Pagandurua, que podría servirle de apoyo ante eventuales acciones defensivas ${ }^{35}$.

Las fortalezas del señor de Agramont protegían precisamente ambas vertientes fronterizas de Ultrapuertos. Por un lado, el castillo de Bidache vigilaba el tránsito fluvial del Bidouze, a la vez que controlaba las parroquias labourdinas más cercanas, de obediencia inglesa. Contaba con una fortaleza con origen en el siglo XII, pero cuyos avatares le llevaron a ser derruida y reconstruida varias veces a lo largo de estos siglos. Por otro lado, esta fortaleza estaba complementada con una infraestructura castral de segundo orden muy cercana, la de $\operatorname{Bardos}^{36}$.

En otro orden de cosas, el castillo de Viellenave-sur-Bidouze constituía un eslabón del cinturón defensivo frente al Béarn. De hecho, aún se pueden observar en ese lugar los restos de una fortaleza ${ }^{37}$ desde la que, según sus contemporáneos, podían divisarse todos los valles de los alrededores, puesto que se localizaba sobre una colina artificial y

33 No ocurría sólo en el caso navarro, sino que también los ingleses prestaban un cuidado especial a la frontera. Eduardo II manifestaba así su interés por mantener en buen estado sus fortificaciones "Ad nostrum parvenit auditum quod in partibus Aquitanie sunt multa castra, domus, maneria et loca de nostris ruinos que reparacione plurimum indigere noscuntur et eciam aliqua inmunita que municione indigent quorumque municio esset perquam utilis nobis et terre" (Charles BÉMONT: Rôles Gascons, tomo IV, p. 122, n 402).

34 Los datos que están compilados a continuación proceden de un texto fundamental para conocer las fortalezas de la zona gascona: Jacques GARDELLES: Les châteaux du Moyen Âge dans la France du Sud-Ouest, ed. Droz, Ginebra, 1972.

35 Se cita específicamente la parroquia de San Esteban de Pagandurua en AGN, Comptos, caj. 5, n 39, II, fol. $6 \mathrm{r}$.

36 Citada en un documento de 1347, en el que se atribuía su guarda, junto a la de Montastruc, a Arnaldo Raimundo, señor de Agramont. (Jean de JAURGAIN La Vasconie. Étude historique et critique, tomo II , Imp. Garet, Pau, 1902, p. 98).

37 Ver la obra de Jean-François-Joseph MASSIE: Le château de Gramont à Viellenave-sur-Bidouze o la de Jean de JAURGAIN: La maison de Gramont, p. 7, que también es citada por Susana HERREROS: Las tierras navarras de Ultrapuertos, p. 81, afirmando que "el actual Viellenave, residencia ya en ese momento de los señores de Agramont". 
estaba rodeado de fosos controlaba toda la cuenca del Bidouze ${ }^{38}$. De esta forma ejercía un control directo sobre la vía fluvial, a la vez que vigilaba los territorios adyacentes, como las parroquias de Came y Escos. Por tanto, los señores de Agramont representaban una pieza fundamental en el esquema de protección de la frontera del monarca navarro, además de tener unos contactos políticos envidiables, de modo que este último buscaría repetidamente su apoyo, con resultados variables. De hecho, los continuos cambios de adscripción de este grupo nobiliario provocaron que en 1329, el rey especulara ya con la posibilidad de que dejaran de incluirse en territorio navarro ${ }^{39}$.

Del mismo modo que la fortaleza agramontesa citada en el párrafo anterior, Luxa formaba parte del cinturón defensivo bearnés y, también se encontraba situada en la ribera de un afluente del Bidouze. De hecho, existían referencias a este castillo ya desde el siglo XI, aunque fue destruido en $1524^{40}$. La fortaleza se entregó en homenaje por Bracs García, señor de Ostabat, Lantabat y Luxa a Teobaldo II en 1258, lo cual garantizaba su disponibilidad en caso de una eventual confrontación con intereses bearneses.

Siguiendo la misma línea fronteriza se puede localizar también la fortaleza de Domezain, detentada por la familia nobiliaria del mismo nombre. Esta infraestructura castral estaba situada en los márgenes de las tierras de Soule y Ultrapuertos, un punto de control fundamental, puesto que a pesar de que el vizconde de Soule se situaba en la obediencia navarra, se encontraba muy comprometido también con sus alianzas inglesas.

De hecho, la fortaleza más relevante de la familia de Soule era la de Mauleón, por la que prestaron homenaje a Teobaldo I en 123441. Esta adscripción navarra generó su asedio a mediados del siglo XIII y la prisión para el propio vizconde. Aún así, esta mota castral serviría al vizconde para presentar batalla unos años más tarde a las fuerzas del senescal inglés. Éste estuvo apoyado por el señor de Sault — que se había comprometido con sus castillos para favorecer la conquista del vizcondado de Soule - y entró en 1257 de nuevo en territorio souletino. Presionado por poderes circundantes, Auger de Soule tuvo que ceder el castillo de Mauleón al monarca inglés, y ya en 1261 este castillo aparecía mencionado como posesión directa del mismo, asignándosele un castellano ${ }^{42}$. Por tanto, la antigua fortaleza adelantada pro-navarra pasó a tener capitanes-castellanos ingleses a finales del siglo XIII, amenazando las tierras ultraportanas.

38 Mateo PARIS: Chronica Majora, tomo V, p. 209: “Montem inaccessibilem, cum castro inexpugnabili quod Egremundus appellatur, ita suis inhabitatoribus destituit, quod omnes qui transibant illac in pace poterant ambulare. Erat enim in rupium cacuminibus turres aedificatae eminebant convallibus. Nec poterant institores vel peregrini nec etiam compatriotae per partes illas transmeare, quin a vispilionibus spoliarentur vel etiam necarentur.(...)"

39 AGN, Comptos, caj. 9, nº 53.

40 Referencia extraída de Manex GOYHENETCHE: Histoire générale du Pays Basque,tomo III, ed. Elkarlanean, San Sebastián, 1999, p. 299 y del Ministerio de Cultura Francés, base Mérimée, ref. PA64000006.

41 Reservando el homenaje ligio prestado al rey de Inglaterra (Ver J. Auguste BRUTAILS: Documents..., p. 7, no VI; AGN, Cartularios, C. 3, p. 164-165).

42 En el PRO, E 101/699/28 aparece un registro de las reparaciones y los suministros para los castillos gascones. 
Si se focaliza la atención en las infraestructuras castrales que se podrían calificar de menor relevancia, a través de la documentación es posible rastrear referencias a fortalezas labourdinas o souletinas como las de San Esteban de Baigorry, Garro, Ezpeleta o Cambo. En primer lugar, San Esteban de Baigorry contaba con una mota castral que era la sede de los vizcondes de Baigorry, dependientes del monarca navarro. Este castillo se denominó también Echauz, una denominación de la cual surgiría a finales del siglo XIII una rama de la antigua familia vizcondal ${ }^{43}$, y contaba con una situación estratégica fundamental, al borde de la Nive de los Alduides y vigilando los pasos pirenaicos secundarios hacia Navarra, a la vez que contrarrestaba las posibles incursiones labourdinas.

Por su parte, la familia de Garro detentaba una fortaleza con el mismo nombre en Mendiondo, que también vigilaba los límites de la tierra de Osses, y en un principio militaban en el bando navarro. Por tanto, el castillo fue asediado y posteriormente arrasado a raíz de los enfrentamientos anglo-navarros por orden del rey de Inglaterra hacia 1244, aunque se reconstruiría poco después. De hecho, a finales del siglo XIII, esta fortaleza aprovechó su carácter fronterizo y desde ella, los señores de Garro comenzaron a atacar intereses navarros, realizando acciones como apropiarse de la madera de los bosques reales de Osses -lo que provocó un enfrentamiento directo con el baile de San Juan de Pie de Puerto- y a los propios señores de Belzunce, situados en una parroquia cercana, Ayherre ${ }^{44}$.

Este espacio fronterizo también se encontraba controlado por varios castillos de titularidad regia - al menos controlados por tenentes del rey-. Por un lado, se podría identificar el de Rocafort ${ }^{45}$, que se encontraba situado en la misma colina que las grutas de Isturitz, un importante asentamiento prehistórico. Dicho castillo controlaba los pasos hacia la tierra de Arberoa y Mixa, una zona en permanente conflicto con los ingleses ${ }^{46}$. Esta fortaleza no sólo interesaba al monarca por su posición geográfica envidiable, sino que a la vez le permitía vigilar los movimientos de la conflictiva nobleza de la zona, ya que prácticamente lindaba con las posesiones de los Garro y a la vez se oponía a la cercana fortaleza de los Belzunce ${ }^{47}$.

Otra de las construcciones que dominaban este espacio fue la de Mondarrain o Monte Ferrandi, y que varios autores localizan en el monte del mismo nombre entre los términos de Ezpeleta e Itsasou ${ }^{48}$. Esta fortaleza fue muy codiciada como punto estratégico por el rey navarro y tras un asedio los señores de Garro se la entregaron en detrimento de los

43 Jean de JAURGAIN: La Vasconie..., tomo II, p. 275.

44 Jean-Baptiste ORPUSTAN: Le château de Garro, capítulo 5. Recurso electrónico consultado el 13/06/ 08 en www.mendionde.fr.gd/le-nom-de-garro.htm

45 Citado en los Comptos reales de 1266, ver Juan CARRASCO: Acta Vectigalia..., tomo I.

46 Su alcaide es asesinado en 1242 por los labortanos: AGN, Comptos, caj. 36, $\mathrm{n}^{\circ} 5$. A través de este mismo texto se sabe que el rey contaba con un tenente localizado allí porque, de hecho, muere en la contienda "Arnald Santz d'Ustubil et Per Arnald d'Arriaga mataron al alcait del castiello de Rocafort cum otro peon" (AGN,

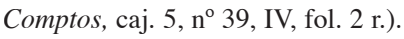

47 Si bien aparece una referencia a las tierras de Garro como tal, y al asedio de su castillo, que se señala en el mapa: "quando obsidio erat apud Garro" (AGN, Comptos, caj. 5, no 39, II, fol. 4r.), aunque no llega a tomarla "predicta domo de Garro non capta” (idem).

48 Según Paul Raymond: Dictionnaire topographique des Pyrénées Atlantiques, p. 115 y otros autores ya citados como Susana HERREROS: Las tierras...p. 150, que lo considera un punto estratégico que vigilaba la entrada al Baztán desde el Labourd. 
intereses del monarca inglés, apareciendo posteriormente en la documentación navarra como tenencia ${ }^{49}$. Esta construcción representaba un puesto de vigilancia en el camino de Labourd, desde Bayona y Ustaritz a Navarra. Dicha fortaleza se oponía y se complementaba, al cambiar de obediencia, con el castillo de Maya, que protegía el valle del mismo nombre, a la sazón atacado también por los ingleses ${ }^{50}$. Además, como ocurría en el caso de Rocafort vigilaba las tierras de los señores de Ezpeleta y Cambo, donde también existían estructuras castrales. Por otro lado, se podía localizar la fortaleza real de Rocabruna, ubicada en Gamarthe, que vigilaba, en este caso, la entrada a través del valle de Ostabares a la zona de Cisa y las localidades de San Juan el Viejo y San Juan de Pie de Puerto ${ }^{51}$. Curiosamente, y como en el caso de Castelrenaut, su importancia para los navarros no llegó más allá de un siglo ${ }^{52}$.

Por último, se pueden citar tres infraestructuras castrales cuya fundación se situaba en torno a mediados del siglo XIV: Castelrenault, Garris y la casa fuerte de la Naupeciada. La primera de ellas debía su curioso nombre del agente real encargado de su construcción, Renaud de Pont. Su primer alcaide se documentaba en 1347 y resulta otro testimonio de la importancia que revestía para Navarra la implantación castral en puntos que controlaran las vías de comunicación. Este castillo se encontraba ubicado en el linde del camino que unía las tierras ultraportanas con Orthez a través de las tierras de Mixa. Además, controlaba también las tierras de Soule, que a mediados del siglo XIV ya se encontraban ocupadas por el castellano de Mauleón, opuesto a los intereses navarros ${ }^{53}$. Sin embargo, esta infraestructura no debió de tener el efecto deseado, ya que al parecer desapareció en poco menos de un siglo ${ }^{54}$. Del mismo modo, la fortaleza de Garris se construyó en $1319^{55}$, dando abrigo a un conglomerado de población en la tierra de Mixa formado por la villa de Garris y la nueva fundación de Saint Palais, cuya importancia venía generada precisamente por el paso de la ruta desde Orthez que se ha mencionado anteriormente. El baile de Mixa residía precisamente en la nueva fortaleza de Garris y se encargaba de su mantenimiento, según lo atestiguaban los comptos reales de 1358 y $1398^{56}$.

Por último, La Naupeciada era una fortaleza relativamente aislada, situada en las cercanías de Hasparren, a cuya construcción militar complementaba. Aparecía mencionada

49 En los registros de 1266 no constan las rentas percibidas por el territorio, pero sí se consigna la tenencia. Ver el estudio introductorio a la obra de Juan CARRASCO: Acta Vectigalia Regni Navarre, tomo I.

50 Una de las pocas referencias al castillo en los Comptos navarros nos dice un siglo después, en 1374: “Ítem, por obras fechas en el castieillo de Mondarrayn, el quoal es en frontera d'Anglaterra (...) seya muyt mal deparado, et que el rey de Casteilla qui venia a poner setio sobre Bayona, las gentes auian a ser derredor del dicho castieillo"(AGN, Comptos, reg. 151, fol. 55v).

51 Susana HERREROS: "El castillo de Rocabruna en Ultrapuertos. Una nueva teoría" en Príncipe de Viana. Anejo, no 14, 1992, p. 381-386.

52 S. HERREROS: Las tierras navarras de Ultrapuertos..., p. 191 citando a J. José MARTÍNENA: Castillos de Navarra, ed. Lancia, 1992, p. 661.

53 Luis Javier FORTÚN: "Las Ordenanzas de Ultrapuertos de 1341” en Príncipe de Viana, n 166, 1981, p. 268.

54 Susana HERREROS: Las tierras navarras de Ultrapuertos..., p. 195.

55 AGN, Comptos, caj. 5, nº 96.

56 Susana HERREROS: Las tierras navarras de Ultrapuertos..., p. 188. 
ya a finales del siglo XIII ${ }^{57}$, aunque los autores han discutido mucho sobre su etimología. En cualquier caso, se seguirá la opinión de Susana Herreros, en tanto que esta denominación se refería al posible naufragio de las barcas en este punto del río Joyeuse ${ }^{58}$. Esta acepción demuestra la importancia geoestratégica de esta fortaleza, ya que se encontraba precisamente vigilando un meandro del río que se ha mencionado, precisamente en la zona en que dicho torrente dejaba de ser navegable, por lo que no sería descabellado pensar que fuera un punto de embarque y desembarque de mercancías. Otro testimonio de su interés geoestratégico lo revela el hecho de que en el mismo punto donde se alzaba la fortaleza los reyes navarros - que pertenecían a la Casa de Francia - decidieron crear una de las escasas bastidas en territorio ultraportano, Labastide Clairence ${ }^{59}$, cuya situación se explicará más adelante.

\section{FUNDACIONES DE VILLAS, BASTIDAS Y ESTRATEGIAS TERRITORIALES}

Con respecto a esta última afirmación se debe puntualizar que para controlar la frontera, los monarcas navarros no sólo establecieron un cinturón de fortalezas, sino que también se apoyaron en la fundación de villas - y el estímulo de su poblamiento-, como en el caso de Saint Palais o de Labastide Clairence. En el primer caso no se conoce la concesión del fuero a la localidad ${ }^{60}$, pero sí se tienen noticias de su estatus privilegiado, o de la prioridad que daba el monarca navarro a su control y defensa, como se explicará a continuación. Esta población se encontraba situada en el extremo superior del apéndice ultraportano, controlando las rutas de comunicación con el Béarn. Se caracterizaba por una cercanía notable respecto a Garris, otra villa fundamental de la zona. Ésta última representaba una fundación antigua ${ }^{61}$, mientras que las primeras referencias de Saint Palais datan de mediados del siglo XIII. Posiblemente esta localidad fuera una fundación navarra relacionada con la necesidad de crear una dicotomía de poderes, oponiendo Garris, la antigua capital vizcondal, a Saint Palais, la villa real navarra - también denominada como Vilanova, indicando su reciente fundación-. Entonces, ¿por qué colocar estas dos villas tan cercanas? La respuesta se encuentra también en la conflictividad latente que representaba la frontera con el Béarn y los problemas ya comentados sobre la titularidad de las tierras de Mixa.

Sin embargo, no se tienen noticias de documentos de fundación o fueros, como se ha comentado aunque, entre otros privilegios, Saint Palais contaba con una ceca durante el siglo XIV, que acuñaba una moneda denominada como pelatus ${ }^{62}$. El monarca recaudaba

57 AGN, Comptos, caj. 321, n 1 , fol. $17 \mathrm{v}$.

58 Susana HERREROS: Las tierras navarras de Ultrapuertos..., p. 198.

59 Aparece citada en los comptos de 1316 "Por la goarda de la casa de la Naupeciada et de la forest de Garharreguia...” y en 1318 se identifica ya con Labastide Clairence "Por la goarda de la casa de la Naupeciada, nichil, por que la bastida de Clarença es fecha en aquel logar" transcritos en Juan CARRASCO: Acta Vectigalia.., tomo X, p. 253 y 436.

60 Susana HERREROS: Las tierras navarras de Ultrapuertos..., p. 176.

61 Paul RAYMOND: Le Cartulaire de l'abbaye de Saint Jean de Sorde, 1983, nº 86, referente a una donación de Español de Labourd.

62 Susana HERREROS: Las tierras navarras de Ultrapuertos..., p. 220. 
en la localidad el cermenaje, lo cual demuestra que si bien era un punto favorecido por el rey, no contaba con las mismas exenciones que otras localidades, como Labastide Clairence. Los habitantes también estaban obligados a moler su trigo en el molino real de San Juan, aunque según ha quedado reflejado en la documentación, no lo hacían, dirigiéndose a molinos señoriales más cercanos ${ }^{63}$. Por otro lado, contaban con un mercado en Garris y con un chapitel en las dos localidades, aunque curiosamente el de Saint Palais no daba beneficios $^{64}$. Además, la aduana situada en esta zona tasaba el tráfico de mercancías, pero también revelaba un volumen notable de contrabando con la zona bearnesa ${ }^{65}$, como ocurría también en otras fronteras europeas.

A través de la documentación se puede observar cómo los monarcas navarros encargaron a sus representantes en la zona la protección de Saint Palais frente a los malhechores de frontera bearnesa, sobre todo a finales del siglo XIV ${ }^{66}$. Al parecer ciertas escaramuzas estaban relacionadas de nuevo con la titularidad contestada de una parroquia, Escos, esta vez entre el rey navarro y el vizconde bearnés ${ }^{67}$. Además de este refuerzo, muy cerca de las dos villas de Garris y Saint Palais se encontraba situado un tercer elemento de control de la frontera, La Población de Mixa, que como su nombre indicaba, representaba también una dependencia directa del rey. Se encontraba fortificada y estaba diseñada como un elemento de apoyo a la infraestructura castral del Garris, ya mencionada. Sin embargo, la situación se volvió tan compleja que en octubre de 1372 el propio rey navarro Carlos II ordenó derribar los muros que protegían el asentamiento, por temor a que cayeran en manos inglesas. Este miedo estaba justificado por un cierto abandono del enclave a favor de la defensa de Garris-Saint Palais ${ }^{68}$, lo cual demostraba aún más su carácter de punto estratégico para el monarca navarro.

En otro orden de cosas, Navarra, precisamente durante el reinado de la Casa de Francia, no quedó fuera de la dinámica de fundaciones de bastidas, representadas por la localidad de Labastide-Clairence. Fue creada en 1312 ante la necesidad de contar con un punto de control en esta zona fronteriza, boscosa y de fácil acceso para los ingleses. Además, se encontraba situada en la orilla del rio Joyeuse, que representaba un hito en el comercio fluvial con Bayona, una situación claramente codiciada por los reyes navarros, que seguían buscando una salida al mar a través de los puertos labourdinos. En el curso del Joyeuse contaban además con el apoyo de la parroquia de Urt, que se había unido a la obediencia navarra.

En cualquier caso, esta posición ya venía marcada por una fundación navarra anterior, una fortaleza ya mencionada, la Naupeciada, que se edificó en torno a $1283^{69}$. Por tanto,

63 Luis J. FORTÚN: Ordenanzas..., p. 271.

64 AGN, Registros, $\mathrm{n}^{\circ} 109$, fol. 248.

65 Susana HERREROS: Las tierras navarras de Ultrapuertos..., p. 284.

66 En concreto, en 1391 envía al mariscal de Navarra, Martín de Lacarra, con hombres de armas para reforzar la villa de Saint Palais, y en 1398 se encargó a Menaud de Sormendi, baile de Mixa que protegiera la villa contra los malhechores de la frontera, que querían atacarles en ausencia del rey (AGN, Comptos, caj. 76, $\mathrm{n}^{\circ}$ 32 , VIII).

67 AGN, Registros, n 202, fol. 263 y 268; AGN, Comptos, caj. 62, nº 17.

68 Ricardo CIÉRBIDE, Javier SANTANO: Colección diplomática de documentos gascones de Baja Navarra, tomo II, ed. Eusko Ikanskuntza, San Sebastián, 1995, p. 201, nº 354.

69 AGN, Comptos, caj. 31, nº 1, fol. 17 v. 


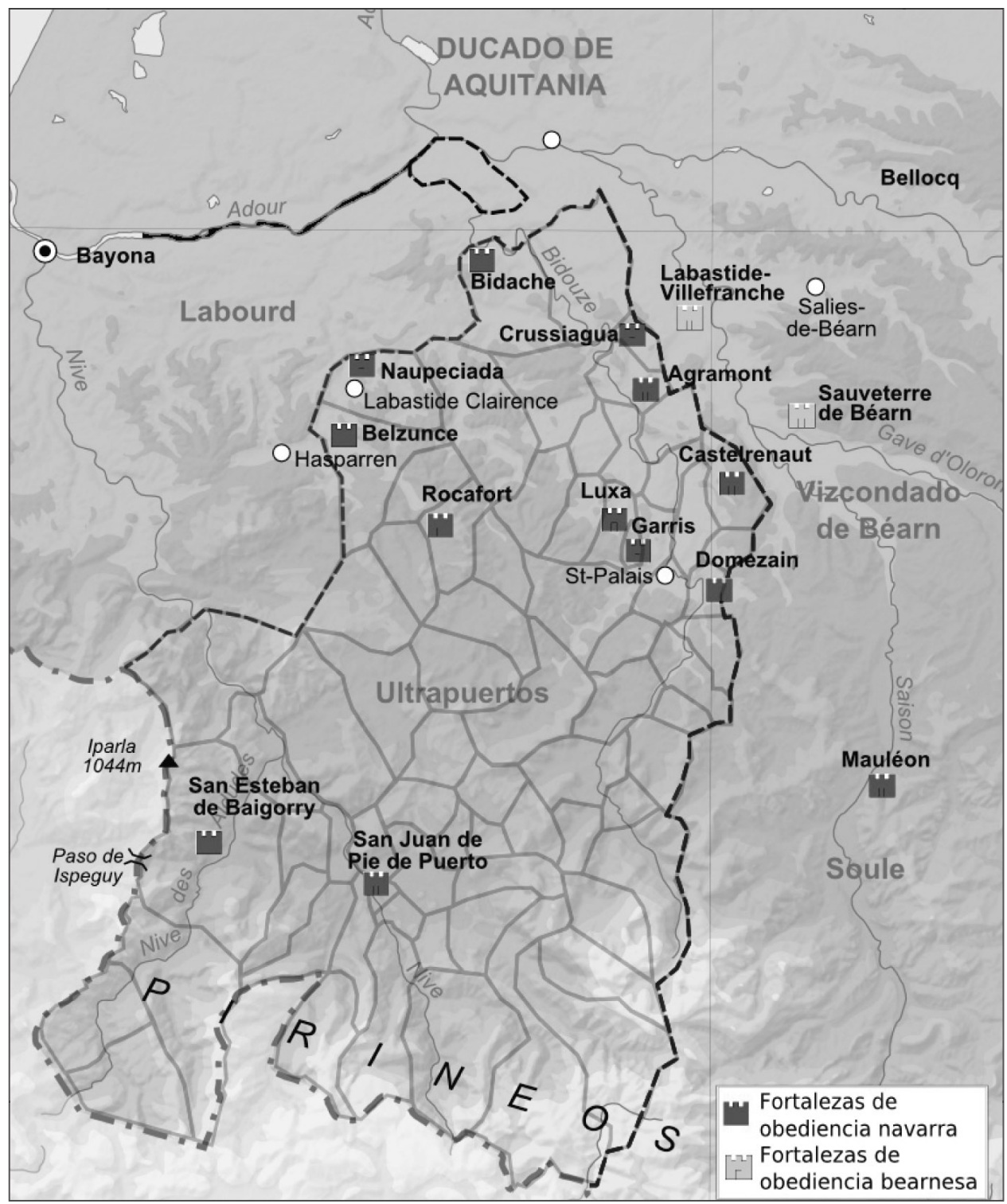

y debido a su importancia geoestratégica, Luis el Huttin decidió fundarla, desgajándola de la zona de Arberoa, y tomando como modelo para su fuero el de la bastida de Rabastens, creada por su padre en Bigorra en 1288. Ya en 1314 se estableció el reparto de los lotes de tierra entre los nuevos pobladores ${ }^{70}$, que además contaban con otros privilegios,

70 AGN, Registros, n 43, fol. 167 v. 
como un mercado semanal los lunes, y dos ferias, en mayo y en San Martín ${ }^{71}$. Su propio fuero ya detectaba la posibilidad de que los comerciantes aprovecharan la zona para pasar fraudulentamente productos extranjeros, por lo que se establecieron ciertas multas ${ }^{72}$. En la misma línea, al otorgárseles los frutos y el agua del bosque cercano de Gaharregia se detallaba la prohibición de comerciar con dichos productos fuera de la propia población ${ }^{73}$.

Por otro lado, se trataba de una zona en conflicto constante, como ocurría en el caso de Saint Palais. En 1357 se vieron enfrentados con la cercana parroquia de Hasparren ${ }^{74}$, mientras que en sus primeros años de andadura tuvieron dificultades hasta en el caso de la construcción de su iglesia, por la que tuvieron que hacer frente a un pleito, durante el cual los testigos afirmaron que al fundarse la bastida se oficiaban las misas en la propia casa del baile, y que construyeron la iglesia con el permiso del mismo baile navarro, aunque no así el del obispo de Bayona, de modo que en 1316 el prelado de Pamplona tuvo que aprovechar un viaje para consagrar dicha iglesia, aunque posteriormente el obispado bayonés reclamó las rentas de este establecimiento ${ }^{75}$.

Sin embargo, la estrategia poblacional del monarca navarro en Labastide no dio los frutos esperados. La localidad recibió habitantes y se desarrolló de modo ascendente hasta 1343, pero a partir de ese momento se estancó y comenzó un notable declive ${ }^{76}$. En 1365 , un informe sobre el despoblamiento de la zona generó nuevas exenciones por parte del monarca navarro ${ }^{77}$, que intuía el peligro que representaba la carencia de habitantes en la zona. El fracaso de este modelo quedó plasmado también en 1392, momento en el que Carlos III, debido a la despoblación, les eximió incluso de su aportación de la ayuda extraordinaria ${ }^{78}$. Por tanto, esta sería una muestra de que pese a los esfuerzos del monarca navarro por controlar las fronteras ultraportanas, sus estrategias no siempre generaban los resultados deseados.

\section{BALANCE FINAL}

Historiográficamente hablando, se ha venido insistiendo constantemente en el carácter permeable de las fronteras pirenaicas, defendiendo que los valores netamente geográficos no resultaban suficientes para definir una realidad tan compleja ${ }^{79}$. Es necesario recor-

71 J. Auguste BRUTAILS: Documents de la Chambre de Comptes de Navarre, doc. XXV, art. 34 y 45.

72 J. Auguste BRUTAILS: Documents..., doc. XXV, art. 18 y 39.

73 AGN, Comptos, caj. 6, nº 9; AGN, Registros, no 5, fol. 102.

74 Susana HERREROS: Las tierras navarras de Ultrapuertos..., p. 203.

75 En concreto, en 1331, según el documento de AGN, Comptos, caj. 31, nº 3 y el de AGN, Códices, C. 7, p. 101-104, aunque en 1347 el propio rey de Navarra le otorgó al obispo dichas rentas para zanjar la situación.

76 Susana HERREROS: Las tierras navarras de Ultrapuertos..., p. 222 y AGN, Registros, n 59, fol. 313.

77 J. Auguste BRUTAILS: Documents de la Chambre de Comptes de Navarre, doc. 102 y la consiguiente exención en AGN, Comptos, caj. 20, n 34.

78 AGN, Comptos, caj. 67, n 35 , XIV.

79 Pierre BONNASSIE: "Introduction" en Philippe SÉNAC (dir.): Frontières et espaces pyrénéens au Moyen Âge, p. 9. o en artículos como el de Charles HIGOUNET: "Une carte des relations monastiques transpyrénéennes au Moyen Âge" en Revue de Comminges, tomo VII, 1962; o Edouard LAMBERT: "Les relations entre la France et l'Espagne par les routes des Pyrénées occidentales au Moyen Âge" en Mélanges D.Faucher, tomo I, Toulouse, 1948, p. 319-328; por poner algunos ejemplos. 
dar, por otro lado, que entidades medievales como el condado de Barcelona, el propio reino de Navarra o las diócesis de Dax y de Bayona desbordaron los limites pirenaicos para extenderse a ambos lados de la cadena montañosa. Precisamente por esta razón es interesante el estudio de la frontera ultrapirenaica, porque si bien constituía una realidad diferenciada del resto de la zona peninsular, contaba, como todos los territorios fronterizos, con espacios de difícil definición e intentos de control constante por parte de los monarcas implicados.

Por tanto, a través de este artículo se ha podido comprobar que, a diferencia de otras fronteras, como la castellano-lusa ${ }^{80}$, los monarcas navarros eran conscientes de la importancia que revestía el enclave ultraportano, y a la vez eran capaces de vislumbrar los posibles peligros que comportaba esta situación de frontera con los ingleses y los bearneses, tanto desde un punto de vista militar como político o fiscal - evasión de mercancías - , y trataron de atajar estos problemas con todos los medios a su alcance.

De ese modo, los reyes navarros y sus agentes establecieron varios niveles distintos de control de esta zona. Por un lado, se apoyaron en las fortalezas reales o nobiliarias asociadas por su homenaje para vigilar los límites del reino, tanto en su vertiente inglesa como bearnesa. Por otro lado, las villas de Labastide, en la zona labourdina, y Saint Palais, en el extremo bearnés, reflejaban los esfuerzos de los monarcas navarros por crear núcleos de poblamiento estable y seguro, atrayendo a los posibles pobladores con diferentes tipos de exenciones. También mostraban un especial cuidado en establecer unas demarcaciones administrativas que les permitieran estructurar el territorio, a la vez que favorecían el sistema recaudatorio y la ejecución de la justicia en Ultrapuertos, un papel fundamental que constituía el pilar de la imagen de dominio del soberano.

En otro orden de cosas, los establecimientos religiosos navarros, de los cuales se ha citado en este artículo el ejemplo de Roncesvalles, también contribuyeron a definir los límites del territorio ultraportano y a fijar la población del mismo, como ocurrió en la encomienda de Bidarray.

Por tanto, debemos decir que en este artículo no se está tratando un tema novedoso, todos conocemos la constante indefinición de las realidades fronterizas medievales. Y es precisamente esta concepción de realidades distintas la que hace este estudio necesario como elemento de comparación, ya que si bien el concepto frontera podía resultar ambiguo, las soluciones aplicadas para controlar dichas zonas resultan tan diversas como interesantes.

\section{DOCUMENTACIÓN DE APOYO}

PRO, SC 8/338/E1244

A nostre seigneur le rei et a son conseil inustre Garsie Arnaud, seigneur de Spelet, que par la ou la paroche de Aigno, que est en la marche de Gascoigne de Navarre, la quele paroche des genz de Navarre de cele marche dient que est de leur seiginerie et les genz

80 Rui CUNHA: "O jogo de escalas hispano-português" en Identidad y representación de la frontera en la España medieval (siglos XI-XIV), ed. Casa de Velázquez, Madrid, 2001, p. 75-88. 
nostre seigneur le rei de de La Burde et de cee marche en Gascoigne dient que la dicte parochie est de la seigneurie nostre seigneur le rex et le rei quant il fust dreni a Burdeus assigna per sa lettre patente le dist Garsy de enquere par assent de gent de Navarre le quel la dite parrochie fust de sa seignurie ou de la seignurie de Navarre, e par mesmes lettres maunda a totz ses officiaus, baillis e autres ministres cele parties que an dist Garsy en ceste chose fusen entendentz e obeisainz issint quant que cele enqueste fust fete qu'el fust maunde au seneschal de Gascoigne le dist Garsy tele lettre par di ovesques ses autres bens en tens de la guerre en la ville de Clyon dinte le dist Garsy prie a nostre seigneur le rei qu'il li goille si ly plest fare encore iccele lettre patente d'enquete, car si la chose ne seit enquise il tourne a grant providite du rei et a grant damage du dist Garsy, a qui nostre seigneur le rei ad grante celes lettres a fee. Et le dist Garsy content qu cele lettre ne fust nenc envoie en Chancelerie ne qu'el ipnse estre trové.

AGN, Comptos, caj. 75, no 38, fol. 2 r. (28/05/1397)

Gil Garcia d'Ianitz, seynor d'Otacu, logartenient de gouernador en Navarra, por el seynnor infant, logartenient del gobernador en el dicto regno, al baille de la tierra de Micxa,et a todos los abitantes en eilla et a quoantos las presentes letras verán et odran en el dicto regno, salut et amor, como ante d'agora nos ouiessemos fecho hun mandato que es en la seguient forma:

Gil Garcia d'Ianitz, seynor d'Otacu, tenient logar del gobernador de Navarra por el seynnor infant, tenient logar de rey en el dicto regno, al baille de la tierra de Micxa, et a todos los abitantes en eilla et a quoantos las presentes letras verán et oyran en el dicto regno, salut et amor. De partes del nobles seynnor de Agramont, por manda de suplication nos es dado a entender que a los abitantes de la villas de Bidaxen, de Reyti, et de Burguey, qui son del regno de Navarra, cada que an menester levar a las dictas villas de la tierra de Micxa trigo, avena, vino o pomada et de las otras viandas necesarias, los embargaden et estorbaden, non leissando lon levar bien, assi como si fuesen extranjeros del regno. Pidiendo non por inde que pues eillos son del regno, que nos demos levar a las dictas villas de las dictas viandas pora provision de ssi et de los abitantes, nin levados ni faziendo levar fuera del regno.

Por ende, nos, inclinado a su suplication vos mandamos firmement et a cada uno de vos que a los abitantes de las dictas villas de Bidaxen, de Reyti et de Burguy, et a cada uno d'eillos dexaden levar trigo, vino, avena, pomada et de las viandas necesarias por provission de ssi et de los viandantes de la dicta tierra de Micxa, de los otros logares de la dicta tierra d'Aillent Puertos que son del dicto logar, segont vsado et acostumbrado han ata agora, faziendo jura que non lievan fuera del dicto regno ni lo faran levar, et non las embarguedes nin los achaquiedes d'aquí adelant contra el thenor d'este nuestro mandamiento, et non falga.

Datum en Pamplona, XII ${ }^{\circ}$ dia de mayo, el tenient logar lo mando. Testigos: l'avat d'Arteiz, don Miguel Periz, don Johan d'Enecuesa, alcaldes. Nota Garcia Periz d'Aranguren. Anno domini $\mathrm{M}^{\mathrm{o}} \mathrm{CCC}^{\mathrm{o}}$ lo sexto.

81 Vos] ms. interlineado. 
Et como agora de parte del dicto seynor d'Agramont et de los abitantes de las dictas villas, suplicando nos sea dado a entender que $\operatorname{vos}^{81}$ o alguno de vos maliciosament veniendo contra el dicto nuestro mandamiento et menospreciando aqueill non los dexedes levar del as dictas viandas, antes los embarguedes, que non lieva, la quoal cosa nos pesa de coraçon, porque conde cabo vos mandados firmement, so pena de cuerpos et bienes, que vos et cada uno de vos dexedes a los de las dictas villas de Bidaxen, de Reyti et de Berguy levar trigo, avena, vino, pomada et de las otras viandas necesarias a las dictas villas, faciendo la dicta jura que non levaran ni faran levar fuera del regno, justa el thenos del dicto nuestro mandamiento, sin embargo nin contrasto alguno, et non falga.

Datum en Olit, XXIIII ${ }^{\circ}$ dia de abril, el dicto thenient logar de gobernador lo mando. Testigos: l'avat d'Arteiz, don Miguel Periz de Leoz, cavaillero, don Johan Periz d'Esparça et don Johan de Necuesa, alcaldes. Nota Johan d'Othoni. Anno Domini $\mathbf{M}^{\mathrm{o}} \mathrm{CCC}^{\circ}$ lo séptimo.

Soppiendo por cierto que si vosotros esto non fazerades por la desobediencia procediremos contra vos en tal mande que vos seiades puynidos. Notum quo supra reddi litteras.

Nos, rey sobredicto, queriendo que las cosas en las sobredictas letras convenidas sean observadas et goardadas, et oviendo aqueillos agradables de nuestra gracia especial et autoridat real aqueillas loamos, retificados et aprobamos. Et por thenor de las presentes confirmamos salvo nuesto drecho en otras cosas et en todas es allano. En testimonio d'esto nos avemos fecho sieillar las presentes de nuestro grant sieillo.

Datum en San Palay, XXVIII ${ }^{\circ}$ dia de mayo, l'aynno de gracia mil $\mathrm{CCC}^{\mathrm{o}}$ novanta et siete.

Ay interlineo do dizen a la Santa Maria de miey aost. Au castet de Sent Johan, et per $\mathrm{XX}$ sueldos de morlanes. Datum ut supra, Johan Çeyludo et por el rey en su consseillo vos presente Johan Coilludo. 